\title{
Mid-infrared Photodetection Using Cyclotron Resonance in Graphene/h-BN van der Waals Heterostructures
}

\author{
Momoko Onodera, ${ }^{1}$ Kei Kinoshita, ${ }^{1}$ Rai Moriya, ${ }^{1}$ Satoru Masubuchi, ${ }^{1}$ \\ Kenji Watanabe, ${ }^{2}$ Takashi Taniguchi, ${ }^{2}$ and Tomoki Machida ${ }^{1 *}$ \\ ${ }^{1}$ Institute of Industrial Science, University of Tokyo, \\ 4-6-1 Komaba, Meguro, Tokyo 153-8505, Japan \\ ${ }^{2}$ National Institute for Materials Science, \\ 1-1 Namiki, Tsukuba 305-0044, Japan \\ (Received May 27, 2019; accepted May 30, 2019)
}

Keywords: graphene, cyclotron resonance, photo-thermoelectric effect, mid-infrared light, photovoltaic effect, van der Waals heterostructures

Charge carriers in graphene, i.e., massless Dirac fermions, form a unique sequence of Landau levels at high magnetic fields, and the cyclotron resonance in graphene is distinctly different from that in two-dimensional electron gas (2DEG) systems based on conventional semiconductors. The energy interval of Landau levels in graphene is much larger than that in 2DEG systems and dependent on the Landau-level index. The selection rule for optical transitions is also different in graphene. Thus, the cyclotron resonance absorption in graphene has attracted considerable interest for developing ultrasensitive detectors of electromagnetic waves ranging from mid-infrared light to $\mathrm{THz}$ waves. Here, we review our earlier experiments on the detection of cyclotron resonance absorption in graphene/hexagonal boron nitride van der Waals heterostructures. In particular, we discuss the development of the experimental detection scheme of cyclotron resonance in graphene utilizing the (i) bolometric, (ii) photovoltaic, and (iii) photo-thermoelectric effects.

\section{Landau Quantization and Cyclotron Resonance in Graphene}

Monolayer graphene has a unique band structure called a Dirac cone with a linear energy dispersion near the K point, which enables us to study the quantum transport of Dirac fermions in solids. ${ }^{(1)}$ By applying a strong perpendicular magnetic field $B$, the energy state of graphene is quantized into Landau levels. The energy of Landau levels in monolayer graphene is expressed as

$$
E_{N}=\operatorname{sgn}(N) v_{F} \sqrt{2 e \hbar|N| B},
$$

where $v_{F}$ denotes the Fermi velocity in graphene, $e$ the elementary charge, $\hbar$ the reduced Planck constant, and $N$ the Landau-level index. On the other hand, the energy of Landau levels in a

*Corresponding author: e-mail: tmachida@iis.u-tokyo.ac.jp

https://doi.org/10.18494/SAM.2019.2445 
two-dimensional electron gas (2DEG) system based on a conventional semiconductor such as $\mathrm{AlGaAs} / \mathrm{GaAs}$ heterostructures is expressed as

$$
E_{N}=\hbar \frac{e B}{m^{*}}\left(N+\frac{1}{2}\right)
$$

where $m^{*}$ is the effective mass of electrons. Figures 1(a) and 1(b) show the Landau levels of Dirac fermions in monolayer graphene and the Landau levels of electrons in GaAs, respectively. There are distinct differences in Landau quantization between graphene and conventional 2DEG systems. The energy of the Landau levels in graphene is proportional to $\sqrt{B}$, and the energy separation between adjacent levels depends on the Landau-level index. The uniqueness of Landau quantization in graphene gives rise to unique cyclotron resonance in graphene as described below.

When graphene is irradiated with light/electromagnetic waves, cyclotron resonance absorption occurs under a condition where the photon energy of illuminated light is equal to the Landau-level separation, and the electrical transport of graphene is modified by the cyclotron
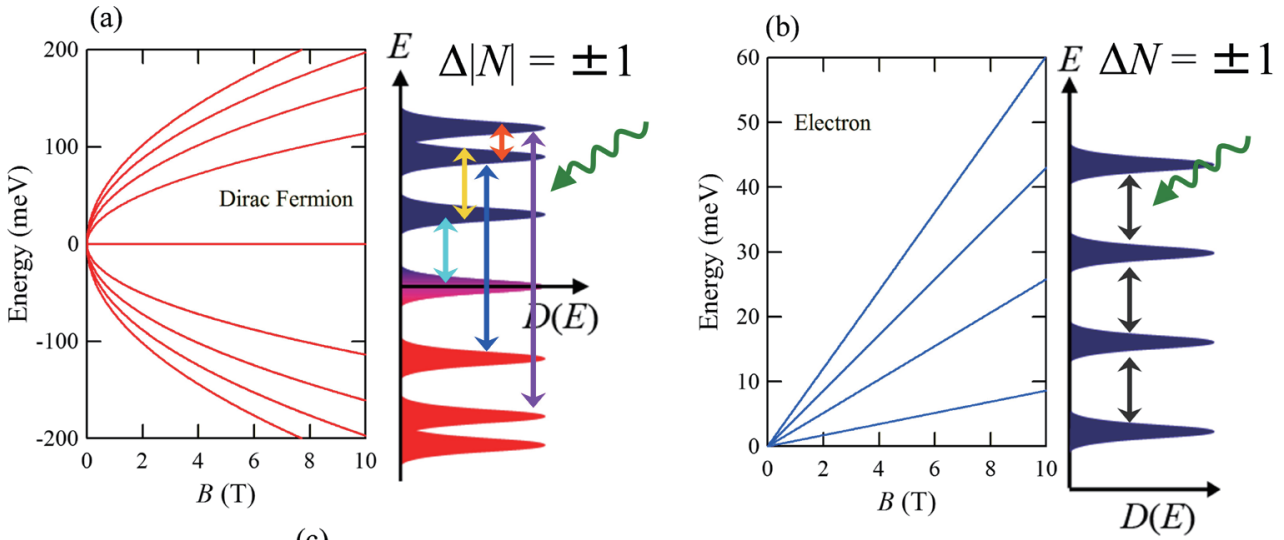

(c)

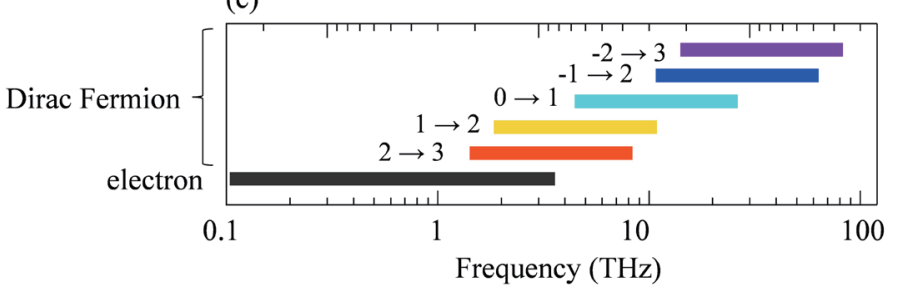

Fig. 1. (Color online) (a) Landau-level energy and schematic density of states in monolayer graphene. The Landau-level separation depends on the Landau-level index. Arrows indicate possible optical transitions induced by cyclotron resonance absorption with an optical selection rule $\Delta|N|= \pm 1$. (b) Landau-level energy and schematic density of states in 2DEG based on the AlGaAs/GaAs heterostructure. The Landau-level separation is independent of the Landau-level index. Arrows indicate possible optical transitions induced by cyclotron resonance absorption with an optical selection rule $\Delta N= \pm 1$. (c) Cyclotron resonance frequency of Dirac Fermions in graphene and that of electrons in $2 \mathrm{DEG}$. 
resonance. This effect can be utilized for developing photodetectors. Indeed, cyclotron resonance in conventional 2DEG systems based on the $\mathrm{AlGaAs} / \mathrm{GaAs}$ heterostructure has already been used for developing quantum-Hall THz-wave photodetectors ${ }^{(2,3)}$ with ultrahigh sensitivity and quantum-dot single-photon detectors. ${ }^{(4)}$

In the case of cyclotron resonance in graphene, first, the selection rule of the photon absorption process is described as $\Delta|N|= \pm 1$, whereas it is $\Delta N= \pm 1$ in conventional 2DEG systems as schematically shown in Figs. 1(a) and 1(b), respectively. Even the optical transition from the hole side to the electron side is allowed. In addition, the energy interval of Landau levels is different depending on the Landau-level index. These features enable photodetection in a wide wavelength range. Secondly, the energy interval of Landau levels in graphene is much larger than that in conventional 2DEG. As a result, the cyclotron resonance energy is in a wavelength range from mid-infrared to near-infrared [Fig. 1(c)], which is technologically important for optoelectronic applications. Thus, cyclotron resonance in graphene has potential advantages for developing ultrasensitive quantum-Hall photodetectors and quantum-dot singlephoton detectors.

\section{Fabrication of Graphene/h-BN van der Waals Heterostructures}

Mechanical exfoliation has been a method of fabricating graphene of the highest quality, although the method appears very simple and primitive. Nevertheless, the inherent quality of graphene is obscured on a $\mathrm{SiO}_{2} / \mathrm{Si}$ substrate because the charged impurities, surface roughness, and optical phonons of $\mathrm{SiO}_{2}$ induce extrinsic carrier scattering into graphene. ${ }^{(5)}$ One of the solutions is to transfer graphene onto hexagonal boron nitride (h-BN) ${ }^{(6)}$ which is a layered material that can be exfoliated similarly to graphite. The exfoliated h-BN flake with an atomically flat surface has the following advantages as a substrate for graphene: the lattice mismatch between h-BN and graphene is less than $2 \%$, the density of charged impurities is extremely low, and the optical phonon energy is higher than that of $\mathrm{SiO}_{2}$. Thus, extrinsic carrier scattering is significantly suppressed in graphene/h-BN, exhibiting intrinsic ultrahigh carrier mobility and intrinsic physics of graphene. ${ }^{(7-9)}$

There are several methods of fabricating graphene/h-BN van der Waals heterostructures (Fig. 2), namely, (i) polymer transfer method, ${ }^{(10)}$ (ii) stamping method, ${ }^{(1)}$ and (iii) autonomous robotic fabrication. ${ }^{(12)}$ In the polymer transfer method, ${ }^{(10)} 2 \mathrm{D}$ materials are exfoliated onto the polymer sheet, which is spin-coated on a $\mathrm{SiO}_{2} / \mathrm{Si}$ substrate, and then crystal flakes are transferred onto another substrate utilizing the thermoplasticity of the polymer. The recently developed polymer-transfer method using polypropylene carbonate (PPC) has great design flexibility and high yields while maintaining high device quality. ${ }^{(13)}$ The stamping method ${ }^{(11)}$ is a technique of picking up 2D crystal flakes using van der Waals force between the 2D crystal flakes. Recently, the autonomous robotic fabrication system has been developed as a next-generation fabrication system composed of the automatic searching of 2D flakes on a $\mathrm{SiO}_{2} / \mathrm{Si}$ substrate and robotic assembly of van der Waals heterostructures. ${ }^{(12)}$ The system is operated automatically and enables us to fabricate van der Waals superlattices of $\sim 30$ layers. 


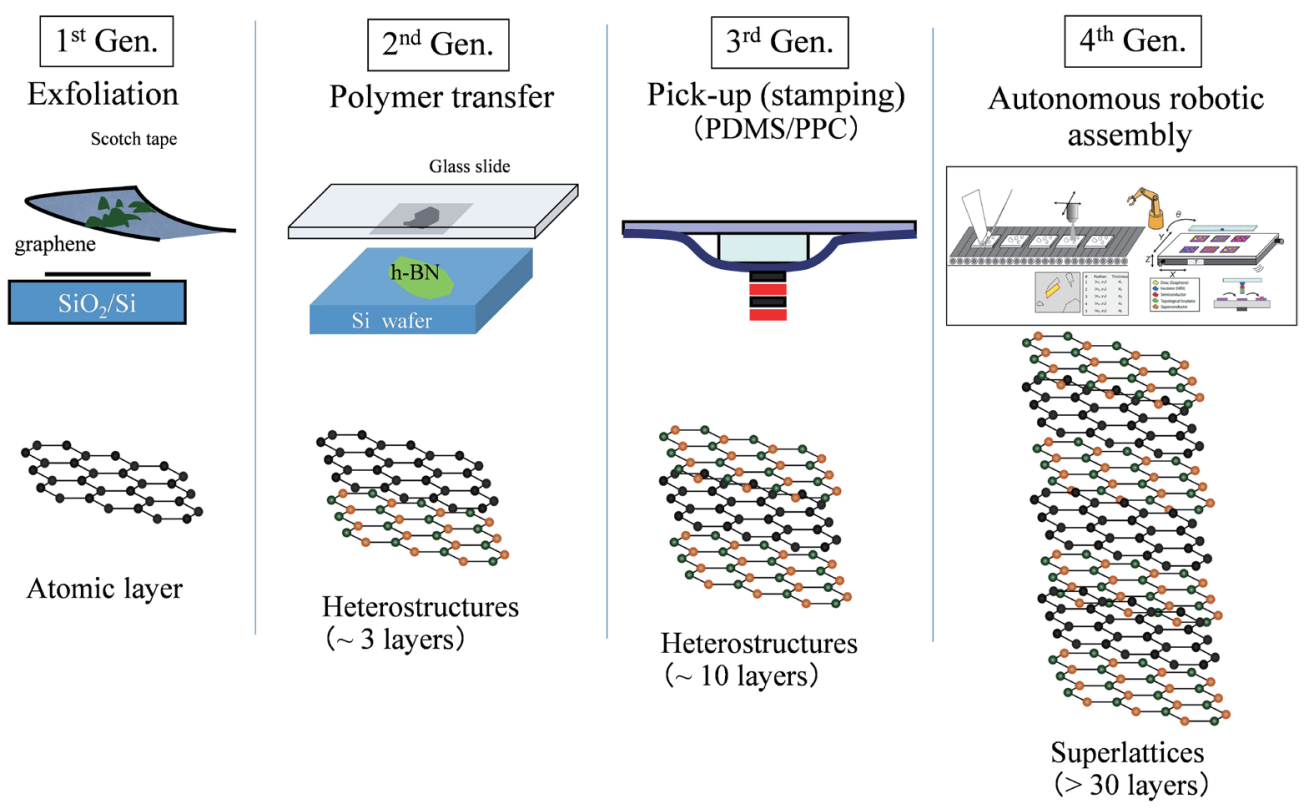

Fig. 2. (Color online) Schematic summary of fabrication methods of van der Waals heterostructures. 1st generation: The mechanical exfoliation technique uses Scotch tape to exfoliate graphite into monolayer graphene. 2nd generation: The polymer transfer technique ${ }^{(10)}$ stacks atomic layers one by one using the thermoplasticity of the polymer. The recently developed PPC dry-transfer method ${ }^{(13)}$ is suitable for fabricating complex structures such as embedded electrodes. 3rd generation: The stamping technique ${ }^{(11)}$ picks up atomic layers by van der Waals force between two crystal flakes of 2D materials. 4th generation: In autonomous robotic assembly, ${ }^{(12)}$ atomic layers are found and stacked by the robotic system, reducing the time and effort for fabrication. This system enables us to fabricate van der Waals superlattices of $\sim 30$ layers.

\section{Cyclotron Resonance Absorption in Graphene/h-BN vdW Heterostructures}

\subsection{Bolometric and photovoltaic effects}

In this subsection, we review our earlier experiments on the detection of cyclotron resonance using the bolometric and photovoltaic effects in graphene/h-BN. ${ }^{(14)}$ A sample of a graphene/ h-BN heterostructure was fabricated using the mechanical exfoliation and polymer transfer technique. A stack of crystal flakes was etched into a Hall-bar shape by electron-beam lithography and plasma etching. Mid-infrared light of $10.6 \mu \mathrm{m}$ wavelength was irradiated onto the device under a strong magnetic field up to $B=9 \mathrm{~T}$. The light was irradiated from a $\mathrm{CO}_{2}$ laser, traveling through an optical fiber and a light pipe to the Hall-bar device at $T=1.5-300 \mathrm{~K}$ in a variable- temperature insert. The laser light was modulated by an optical chopper, and the photovoltage was measured by the lock-in technique.

The electronic state in graphene is quantized into Landau levels at a perpendicular magnetic field of $B=8 \mathrm{~T}$, exhibiting the quantum Hall effect of Dirac Fermions at the Landau filling factors $v=2,6,10, \ldots$, where the longitudinal resistance and Hall resistance are quantized into $R_{x x}=0$ and $R_{x y}=\hbar / 2 e^{2}, \hbar / 6 e^{2}, \hbar / 10 e^{2}, \ldots$, respectively [Fig. 3(a)]. When mid-infrared 

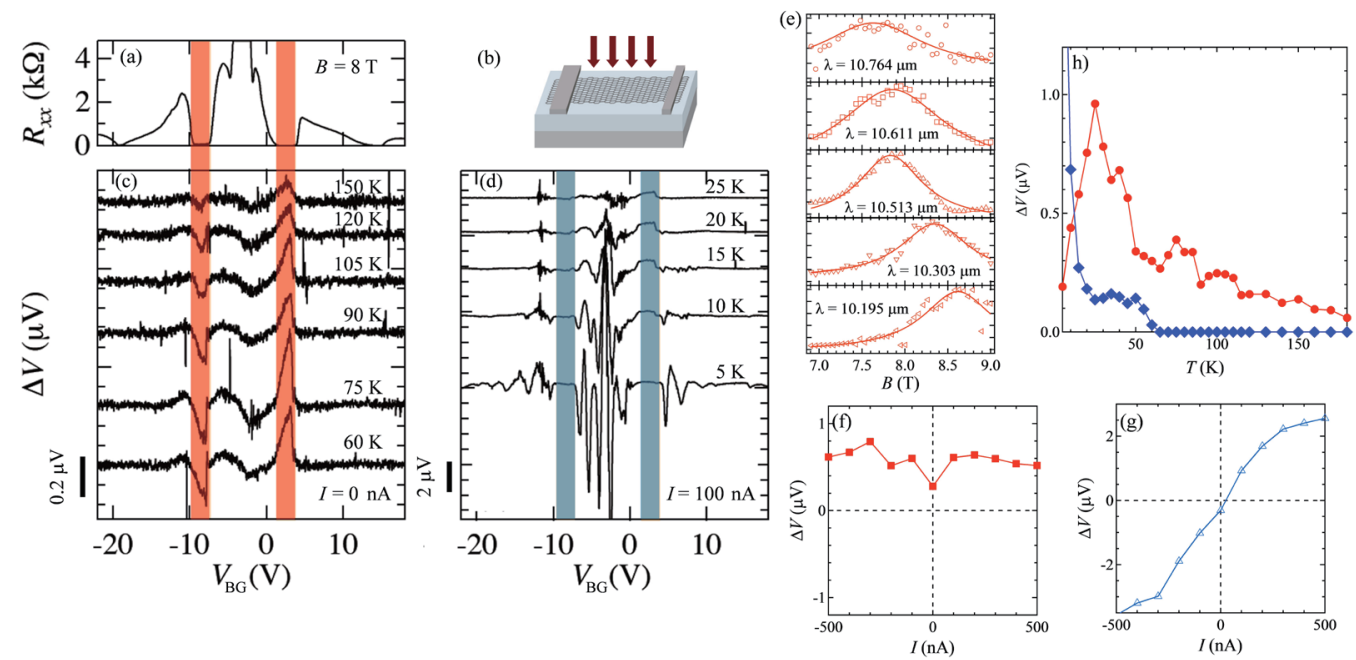

Fig. 3. (Color online) (a) Longitudinal resistance as a function of back-gate voltage $V_{B G}$ measured at $T=25 \mathrm{~K}$ and $B=8 \mathrm{~T}$. (b) A schematic of graphene Hall-bar device. (c) Photoresponse measured with $I=0 \mathrm{nA}$ induced by irradiating mid-infrared light of $10.6 \mu \mathrm{m}$ wavelength. Each curve is offset for clarity. The colored-strip areas indicate $v= \pm 2$ quantum Hall regime. (d) Photoresponse measured with $I=100 \mathrm{nA}$ induced by irradiating midinfrared light of $10.6 \mu \mathrm{m}$ wavelength. (e) Photoresponse as a function of magnetic field for various incident light wavelengths with the photon energy of $115-122 \mathrm{meV}$ (from top to bottom). (f) Photoresponse at $v=2$ quantum Hall regime as a function of $I$ at $T=15 \mathrm{~K}$. (g) Photoresponse at $v=1.3$ quantum Hall transition regime. (h) Temperature dependence of the photoresponse measured with $I=100 \mathrm{nA}$ at $v=2.0$ quantum Hall regime (red circles) and at $v=1.3$ quantum Hall transition regime (blue squares). Reprinted with permission from Ref. 14.

light of $10.6 \mu \mathrm{m}$ wavelength was irradiated onto the device [Fig. 3(b)], photoresponse signals were observed at $v= \pm 2$ as shown by colored-strip regions in Fig. 3(c), where the dependence of the photoresponse signal $\Delta V$ on the back-gate bias voltage $V_{B G}$ at $T=60-150 \mathrm{~K}$ and $I_{S D}=0$ is demonstrated. Figure 3(d) shows the dependence of $\Delta V$ on $V_{B G}$ at $T=5-25 \mathrm{~K}$ and $I_{S D}=100$ $\mathrm{nA}$. Photoinduced signals were observed in transition regions between quantum Hall regimes of $v=-6,-2,+2$, and +6 . Two different types of signal were observed: those observed in the quantum Hall regime and those in the quantum Hall transition regime. Although both types of signal coexisted, the former was dominant at high temperatures and low bias currents, whereas the latter was dominant at low temperatures and high bias currents. These experimental results thus suggest the presence of two mechanisms for the observed photoresponse.

The photoresponse in the quantum Hall regime originates from cyclotron resonance absorption as confirmed by wavelength dependence experiments shown in Fig. 3(e), where the resonance magnetic field shifted systematically as the wavelength of the irradiated laser was changed. In addition, the photoinduced signals in the quantum Hall regime were independent of the bias current as shown in Fig. 3(f), i.e., the cyclotron resonance absorption induces the photovoltaic effect in graphene in the quantum Hall regime.

The photoinduced signals in the quantum Hall transition regime, on the other hand, were proportional to the bias current as shown in Fig. 3(g). Thus, the signals were based on the 
bolometric effect, i.e., the resistance was changed by the absorption of mid-infrared light, leading to photoinduced signals proportional to the bias current. Therefore, the photoinduced signals observed in the experiments described in this subsection are expressed as the summation of both signals as

$$
\Delta V=V_{\text {photovoltaic }}+\frac{\partial R}{\partial T} \Delta T \cdot I_{S D}
$$

Figure 3(h) shows the temperature dependence of the photoresponse signal $\Delta V$ at $I_{S D}=100$ $\mathrm{nA}$. The photoresponse induced by the bolometric effect decreased rapidly as the temperature decreased, and no signal was observed at $T>70 \mathrm{~K}$. On the other hand, the photovoltaic effect based on cyclotron resonance was observable even at $T>180 \mathrm{~K}$. This result suggests that it can be used for a high-sensitivity mid-infrared photodetector that can be operated in a practically accessible low-temperature range.

Earlier developments of the quantum-Hall THz-wave photodetectors ${ }^{(2,3)}$ and quantum-dot single-photon detectors ${ }^{(4)}$ realized extremely high detectability by utilizing cyclotron resonance in $2 \mathrm{DEG}$ systems and quantum dots based on the $\mathrm{AlGaAs} / \mathrm{GaAs}$ heterostructure. Unfortunately, however, they require cryogenic temperatures lower than $10 \mathrm{~K}$ or $100 \mathrm{mK}$, respectively. The disadvantages of the operating temperature and the narrow range of detectable wavelengths could be solved by utilizing a quantum-Hall system based on graphene/h-BN van der Waals heterostructures.

\subsection{Photo-thermoelectric effect}

Earlier experiments on photodetection in graphene under zero magnetic field were mainly based on two types of detection mechanism: the bolometric and photovoltaic effects. (i) Bolometric photodetection utilizes the change in resistance of graphene under light illumination, induced by the increase in electron temperature caused by light absorption. (ii) Photovoltaic photodetection utilizes the generation of photovoltage induced by the dissociation of electron-hole pairs due to the built-in potential in graphene. On the other hand, recent studies have shown that the photo-thermoelectric effect is the main source of photoinduced signals in graphene. ${ }^{(15-20)}$ (iii) Photo-thermoelectric detection utilizes the temperature gradient in graphene generated by light absorption. In the presence of a temperature gradient, carriers move from a hot region to a cold one, which generates photovoltage. Since the electron temperature in graphene is very sensitively increased by light absorption because of its small heat capacity, the small density of states, and weak electron-phonon coupling, the photothermoelectric effect gives rise to a large photoinduced voltage in graphene at zero magnetic field. Thus, one can naturally expect that the photo-thermoelectric effect can generate a large photoresponse also under high magnetic fields. In this subsection, we review our recent experiments on the detection of cyclotron resonance using the photo-thermoelectric effect in graphene/h-BN heterostructures at high magnetic fields. ${ }^{(21)}$ 
First, we discuss the scheme of photo-thermoelectric detection of photoresponses by considering a symmetric graphene two-terminal device shown in Fig. 4(a). Under the midinfrared light illumination, the graphene device is heated up, i.e., the electron temperature of graphene is increased owing to light absorption, while the metallic electrodes remain cold. The resulting temperature gradient near the electrodes generates a photovoltage between the graphene device and the electrodes as described as

$$
V_{\text {thermoelectric }}=\int S \frac{\partial T}{\partial x} d x
$$

where $S$ is the Seebeck coefficient and $\partial T / \partial x$ is the temperature gradient in graphene along the conduction channel. In an ideally symmetric two-terminal device, however, the photovoltage generated near the left electrode and that near the right electrode cancel out, without giving rise to a detectable photovoltage between the electrodes.

To detect the photovoltage induced by the photo-thermoelectric effect, we should introduce asymmetry in the carrier density of graphene as schematically shown in Fig. 4(b), where the carrier density of graphene in the right region is much larger than that in the left region. Since the Seeback coefficient depends on the carrier density of graphene as shown in Fig. 4(c), the photo-thermoelectric effect is significantly suppressed in the right region. Then, the photovoltage generated near the left electrode and that near the right electrode do not cancel out, giving rise to a finite detectable photovoltage between the electrodes.
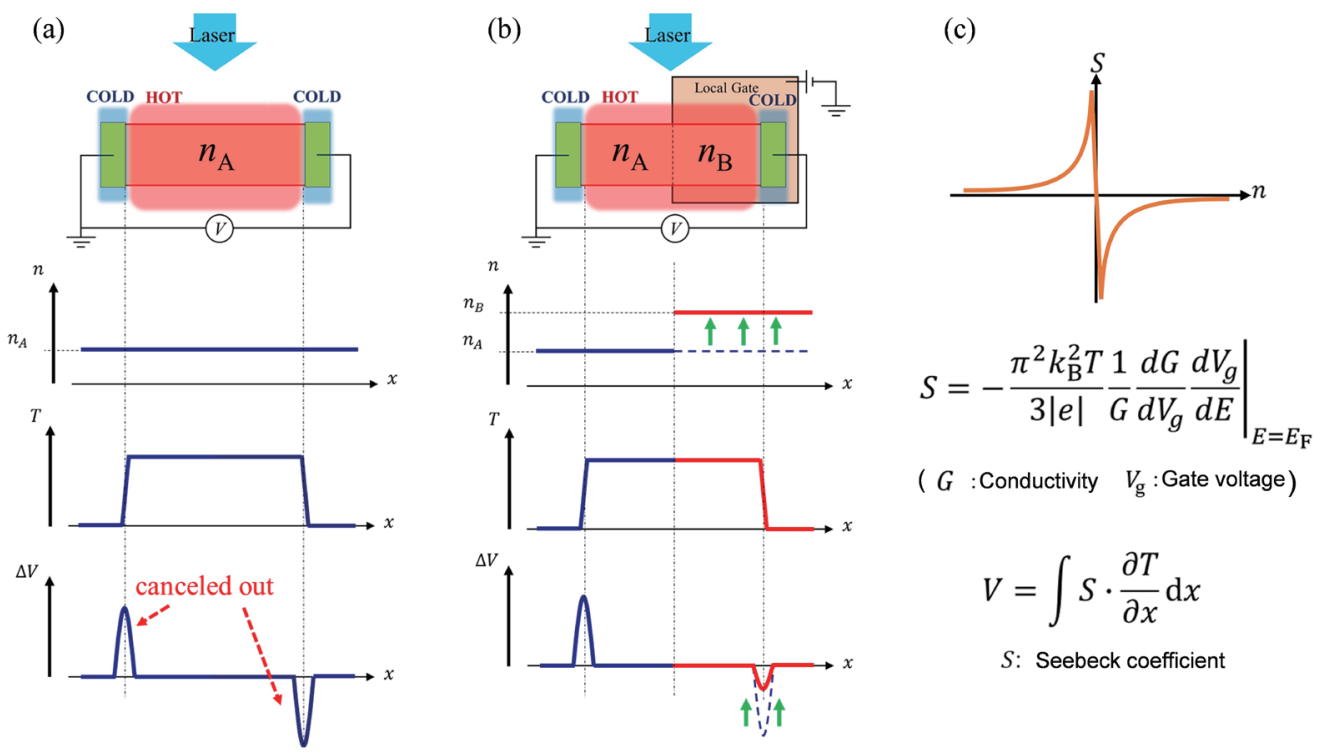

Fig. 4. (Color online) Schematic representations of carrier density, electron temperature, and photovoltage induced by photo-thermoelectric effect in graphene with (a) uniform carrier density and (b) asymmetric carrier density. (c) Dependence of Seebeck coefficient $S$ on carrier density $n$. The value of $S$ reaches its peak and changes its polarity near the Dirac point. 
We realized graphene with asymmetric carrier density by employing the device structure that has a graphite local bottom-gate electrode covering only the right-hand side of graphene [Figs. 5(a) and 5(b)]. In this device structure, the carrier density of graphene on the left and right can be tuned independently by applying different gate-bias voltages to the $\mathrm{p}^{+}$-doped $\mathrm{Si}$ global back gate and the graphite local bottom gate.

The device was irradiated by laser light with a wavelength of $\lambda=10.182 \mu \mathrm{m}$, and the photoinduced voltage $V_{\text {induced }}$ was measured by sweeping the carrier density $n_{L}$ at different magnetic fields $B$ as shown in Fig. 5(c). The amplitude of the signal increased as $n_{L}$ approaches the Dirac point. Furthermore, the sign of the signal reversed at the Dirac point. There are noticeable coincidences between the shape of $V_{\text {induced }}$ [Fig. 5(c)] and that of $S$ [Fig. 4(c)]: (i) the sign is reversed at the Dirac point and (ii) the value approaches zero as the absolute carrier density increases. Thus, it is suggested that the observed photo-response signals originated from the photo-Seebeck component of the photo-thermoelectric effect. Upon application of a magnetic field, $V_{\text {induced }}$ exhibits oscillatory changes with respect to $n_{L}$. This is attributed to the Landau quantization in graphene.

Next, we discuss the magnetic field dependence of $V_{\text {induced }}$ at high magnetic fields. Figure 6(a) shows the magnetic field dependence of $V_{\text {induced }}$ measured under irradiation with light of different wavelengths. We observed the enhancements of the photovoltage signal in low $(\sim 1.5$ $\mathrm{T})$ and high ( $\sim 9 \mathrm{~T})$ magnetic fields. These enhancements are due to the cyclotron resonance absorption of mid-infrared light in graphene. As shown in Fig. 1(a), the cyclotron resonance of the Landau-quantized graphene can absorb light when the selection rule is satisfied. Both the

(a)

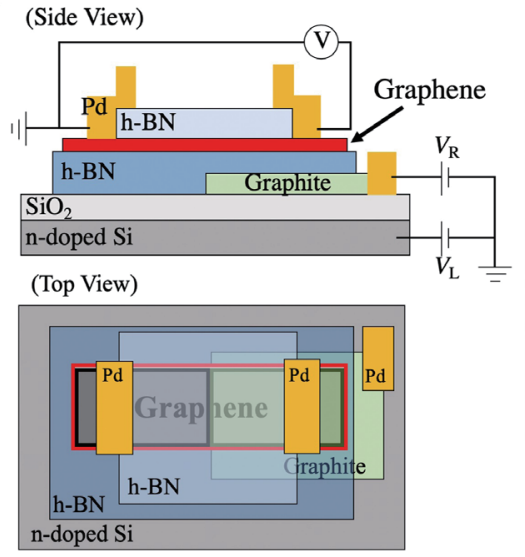

(c)

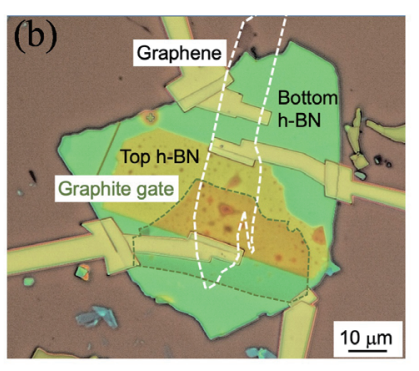

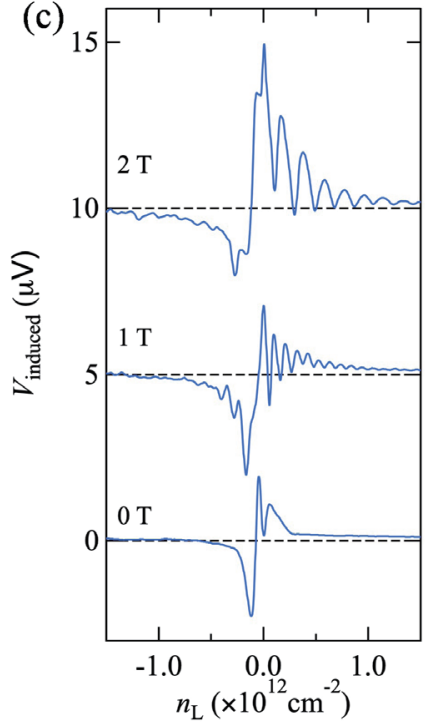

Fig. 5. (Color online) (a) Schematic illustration of the device. Graphene is sandwiched by two h-BN layers; both have a thickness of $\sim 40 \mathrm{~nm}$. Under the bottom h-BN, an exfoliated graphite layer was placed as the local bottomgate electrode. The carrier density $n_{R}$ on the right side of graphene can be changed by applying $V_{R}$ to the graphite local gate, and the carrier density $n_{L}$ on the left side can be controlled by applying $V_{L}$ to the p+-doped Si global back gate. (b) Optical micrograph of the device. (c) Photoinduced voltage $V_{\text {induced }}$ versus $n_{L}$ measured at different magnetic fields $B$. Reprinted with permission from Ref. 21 . 

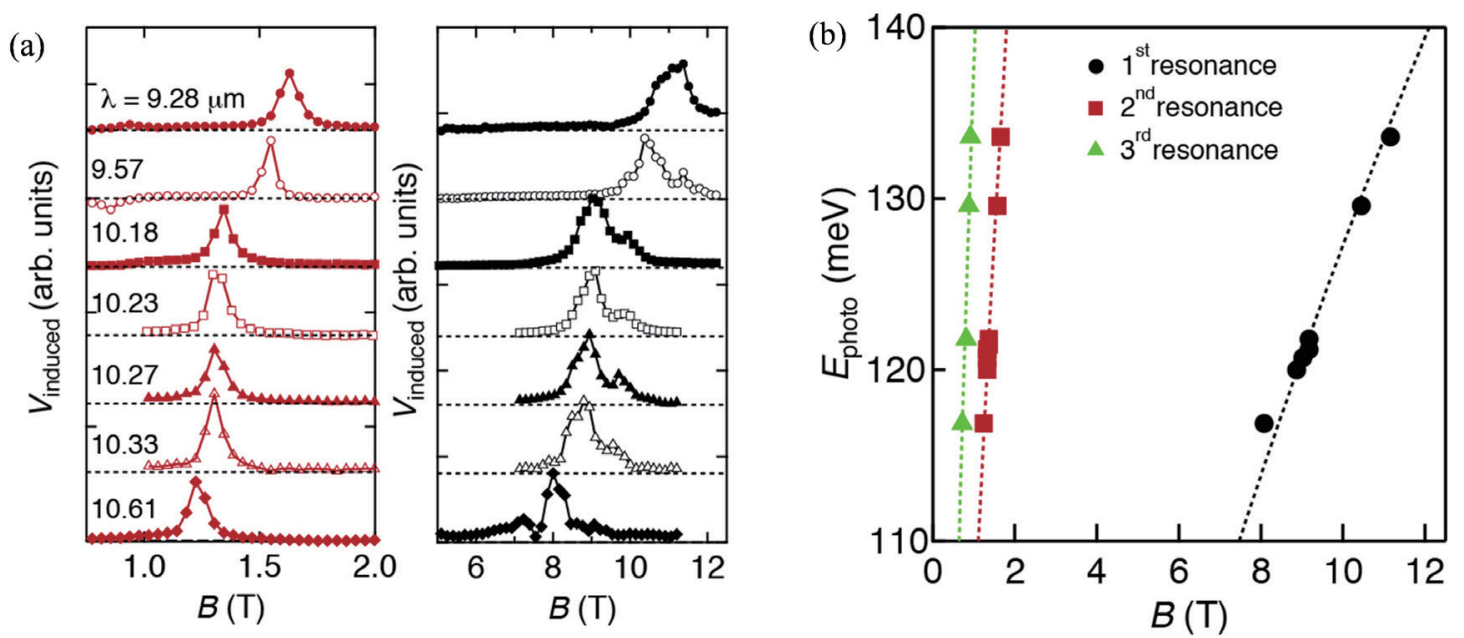

Fig. 6. (Color online) (a) Magnetic field dependence of $V_{\text {induced }}$ measured at different wavelengths. (b) Relationship between the energy of irradiated light and the magnetic field where the cyclotron resonance occurred. Reprinted with permission from Ref. 21.

low- and high-magnetic-field peaks in Fig. 6(a) correspond to the transition of $N=-1 \rightarrow 2$ and $N=-2 \rightarrow 1$ (2nd resonance) and $N=0 \rightarrow 1$ and $N=-1 \rightarrow 0$ (1st resonance), respectively. The relationship between the peak magnetic field values in Fig. 6(a) and the energy of the incident lights are plotted in Fig. 6(b). Here, we also plot other cyclotron resonance peaks $N=-2 \rightarrow N=$ 3 and $N=-3 \rightarrow N=2$ (3rd transition, data is not shown) observed in the same sample. All the resonance fields can be well explained with Eq. (1), which are plotted with dotted lines in the figure. These are additional lines of evidence that the cyclotron resonance of graphene can be detected by the photo-thermoelectric effect.

\section{Acknowledgments}

This work was supported by CREST, Japan Science and Technology Agency (JST) under Grant Number JPMJCR15F3, and by JSPS KAKENHI under Grant Numbers JP19H02542 and JP19H01820.

\section{References}

1 A. K. Geim and K. S. Novoselov: Nat. Mater. 6 (2007) 183. https://doi.org/10.1038/nmat1849

2 Y. Kawano, Y. Hisanaga, and S. Komiyama: Phys. Rev. B 59 (1999) 12537. https://doi.org/10.1103/ PhysRevB.59.12537

3 K. Ikushima, H. Sakuma, S. Komiyama, and K. Hirakawa: Phys. Rev. Lett. 93 (2004) 146804. https://doi. org/10.1103/PhysRevLett.93.146804

4 S. Komiyama, O. Astafiev, V. Antonov, T. Kutsuwa, and H. Hirai: Nature 403 (2000) 405. https://doi. org/10.1038/35000166

5 J.-H. Chen, C. Jang, S. Xiao, M. Ishigami, and M.S. Fuhrer: Nat. Nanotechnol. 3 (2008) 206. https://doi. org $/ 10.1038 /$ nnano. 2008.58

6 C. R. Dean, A. F. Young, I. Meric, C. Lee, L. Wang, S. Sorgenfrei, K. Watanabe, T. Taniguchi, P. Kim, K. L. Shepard, and J. Hone: Nat. Nanotechnol. 5 (2010) 722. https://doi.org/10.1038/nnano.2010.172 
7 S. Masubuchi, K. Iguchi, T. Yamaguchi, M. Onuki, M. Arai, K. Watanabe, T. Taniguchi, and T. Machida: Phys. Rev. Lett. 109 (2012) 036601. https://doi.org/10.1103/PhysRevLett.109.036601

8 S. Morikawa, S. Masubuchi, R. Moriya, K. Watanabe, T. Taniguchi, and T. Machida: Appl. Phys. Lett. 106 (2015) 183101. https://doi.org/10.1063/1.4919380

9 Y. Asakawa, S. Masubuchi, N. Inoue, S. Morikawa, K. Watanabe, T. Taniguchi, and T. Machida: Phys. Rev. Lett. 119 (2017) 186802. https://doi.org/10.1103/PhysRevLett.119.186802

10 A. Castellanos-Gomez, M. Buscema, R. Molenaar, V. Singh, L. Janssen, H. S. J. van der Zant, and G. A. Steele: 2D Mater. 1 (2014) 011002. https://doi.org/10.1088/2053-1583/1/1/011002

11 L. Wang, I. Meric, P. Y. Huang, Q. Gao, Y. Gao, H. Tran, T. Taniguchi, K. Watanabe, L. M. Campos, D. A. Muller, J. Guo, P. Kim, J. Hone, K. L. Shepard, and C. R. Dean: Science 342 (2013) 614. https://doi.org/10.1126/ science. 1244358

12 S. Masubuchi, M. Morimoto, S. Morikawa, M. Onodera, Y. Asakawa, K. Watanabe, T. Taniguchi, and T. Machida: Nat. Commun. 9 (2018) 1413. https://doi.org/10.1038/s41467-018-03723-w

13 K. Kinoshita, R. Moriya, M. Onodera, Y. Wakafuji, S. Masubuchi, K. Watanabe, T. Taniguchi, and T. Machida: Npj 2D Mater. Appl. 3 (2019) 22. https://doi.org/10.1038/s41699-019-0104-8

14 S. Masubuchi, M. Onuki, M. Arai, T. Yamaguchi, K. Watanabe, T. Taniguchi, and T. Machida: Phys. Rev. B 88 (2013) 121402. https://doi.org/10.1103/PhysRevB.88.121402

15 N. M. Gabor, J. C. W. Song, Q. Ma, N. L. Nair, T. Taychatanapat, K. Watanabe, T. Taniguchi, L. S. Levitov, and P. Jarillo-Herrero: Science 334 (2011) 648. https://doi.org/10.1126/science.1211384

16 K. J. Tielrooij, M. Massicotte, L. Piatkowski, A. Woessner, Q. Ma, P. Jarillo-Herrero, N. F. van Hulst, and F. H. L. Koppens: J. Phys. Condens. Matter 27 (2015) 164207. https://doi.org/10.1088/0953-8984/27/16/164207

17 A. Brenneis, F. Schade, S. Drieschner, F. Heimbach, H. Karl, J. A. Garrido, and A. W. Holleitner: Sci. Rep. 6 (2016) 35654. https://doi.org/10.1038/srep35654

18 X. Xu, N. M. Gabor, J. S. Alden, A.M. van der Zande, and P. L. McEuen: Nano Lett. 10 (2010) 562. https://doi. org $/ 10.1021 / \mathrm{n} 1903451 \mathrm{y}$

19 G. Skoblin, J. Sun, and A. Yurgens: Appl. Phys. Lett. 112 (2018) 063501. https://doi.org/10.1063/1.5009629

20 D. A. Bandurin, I. Gayduchenko, Y. Cao, M. Moskotin, A. Principi, I. V. Grigorieva, G. Goltsman, G. Fedorov, and D. Svintsov: Appl. Phys. Lett. 112 (2018) 141101. https://doi.org/10.1063/1.5018151

21 K. Kinoshita, R. Moriya, M. Arai, S. Masubuchi, K. Watanabe, T. Taniguchi, and T. Machida: Appl. Phys. Lett. 113 (2018) 103102. https://doi.org/10.1063/1.5045731 